\title{
Cisto periapical em região anterior da maxila: relato de caso
}

Periapical cyst in the anterior maxilla: case report

Quiste periapical en el maxilar anterior: reporte de caso

Híttalo Carlos Rodrigues de ALMEIDA

Faculdade de Odontologia da Universidade de Pernambuco (FOP-UPE), 50740-540 Recife-PE, Brasil https://orcid.org/0000-0001-7348-6601

Zilda Betânia Barbosa Medeiros de FARIAS

Faculdade de Odontologia da Universidade de Pernambuco (FOP-UPE), 50740-540 Recife-PE, Brasil https://orcid.org/0000-0001-9549-2260

Bruna Peixoto Nogueira dos SANTOS

Faculdade de Odontologia da Universidade de Pernambuco (FOP-UPE), 50740-540 Recife-PE, Brasil https://orcid.org/0000-0003-4068-5887

Rebeka Thiara Nascimento dos SANTOS

Faculdade de Odontologia da Universidade de Pernambuco (FOP-UPE), 50740-540 Recife-PE, Brasil https://orcid.org/0000-0002-0422-8593

Rômulo Oliveira de Hollanda VALENTE

Cirurgião Bucomaxilofacial do Hospital Getúlio Vargas, 50630-060 Recife-PE, Brasil https://orcid.org/0000-0002-2806-9945

Márcia Maria Fonseca da SILVEIRA

Faculdade de Odontologia da Universidade de Pernambuco (FOP-UPE), 50740-540 Recife-PE, Brasil https://orcid.org/0000-0002-2611-5661

Ana Paula Veras SOBRAL

Faculdade de Odontologia da Universidade de Pernambuco (FOP-UPE), 50740-540 Recife-PE, Brasil https://orcid.org/0000-0002-0801-9385

\section{Resumo}

Introdução: O cisto periapical é um cisto de natureza inflamatória que está relacionado ao ápice de um dente com necrose pulpar e corresponde à frequência de $7 \%$ a $54 \%$ das imagens periapicais. Objetivo: Relatar um caso clínico de um paciente com cisto periapical em região anterior da maxila. Relato de caso: Paciente do sexo feminino, 20 anos de idade, compareceu a clínica de Estomatologia do Centro de Pós-graduação em Odontologia, CPGO, com queixa de um discreto aumento de volume na região anterior da maxila há 5 anos. Ao exame extra-oral foi observada discreta assimetria e no exame intra-oral, na região do dente 12 a mucosa apresentava-se com coloração normal e de consistência resiliente. No exame radiográfico foi observado imagem radiolúcida entre as raízes dos dentes 13 e 12. No exame tomográfico foi confirmada a imagem com preservação da cortical óssea. Frente aos aspectos clínico e radiográfico, foram sugeridas as hipóteses diagnósticas de cisto periapical, tumor odontogênico ceratocístico, ou ameloblastoma. A conduta foi a exérese da lesão, seguidas de curetagem e envio do espécime para análise histopatológica, tendo como resultado cisto periapical. Conclusão: O dente 12 foi tratado endodonticamente após o diagnóstico e a paciente está sendo acompanhada para se analisar a formação de osso sadio no local que antes era ocupado pelo cisto.

Descritores: Cisto Periapical; Necrose Pulpar; Maxila.

\section{Abstract}

Introduction: The periapical cyst is an inflammatory cyst that is related to the apex of a tooth with pulp necrosis and corresponds to the frequency of $7 \%$ to $54 \%$ of the periapical images. Objective: To report a clinical case of a patient with periapical cyst in the anterior region of the maxilla. Case report: A 20-year-old female patient attended the Stomatology clinic at the Graduate Center for Dentistry, CPGO, complaining of a slight increase in volume in the anterior region of the maxilla 5 years ago. In the extra-oral examination, a slight asymmetry was observed and in the intra-oral examination, in the region of tooth 12 , the mucosa was normal and resilient in consistency. In the radiographic examination, a radiolucent image was observed between the roots of teeth 13 and 12. In the tomographic examination, the image was confirmed with preservation of the bone cortex. In view of the clinical and radiographic aspects, the diagnostic hypotheses of periapical cyst, keratocystic odontogenic tumor, or ameloblastoma have been suggested. The conduct was the excision of the lesion, followed by curettage and sending the specimen for histopathological analysis, resulting in a periapical cyst. Conclusion: Tooth 12 was treated endodontically after diagnosis and the patient is being followed up to analyze the formation of healthy bone in the place that was previously occupied by the cyst.

Descriptors: Periapical Cyst; Dental Pulp Necrosis; Jaw.

\section{Resumen}

Introducción: El quiste periapical es un quiste inflamatorio que se relaciona con el ápice de un diente con necrosis pulpar y corresponde a la frecuencia del $7 \%$ al $54 \%$ de las imágenes periapicales. Objetivo: Reportar un caso clínico de un paciente con quiste periapical en la región anterior del maxilar. Caso clínico: paciente de 20 años que acude a la consulta de Estomatología del Centro de Graduados en Odontología, CPGO, quejándose de un ligero aumento de volumen en la región anterior del maxilar hace 5 años. En el examen extraoral se observó una ligera asimetría y en el examen intraoral, en la región del diente 12, la mucosa era normal y de consistencia resiliente. En el examen radiográfico se observó una imagen radiolúcida entre las raíces de los dientes 13 y 12. En el examen tomográfico se confirmó la imagen con preservación de la cortical ósea. En vista de los aspectos clínicos y radiográficos, se han sugerido las hipótesis diagnósticas de quiste periapical, tumor odontogénico queratoquístico o ameloblastoma. La conducta fue la exéresis de la lesión, seguida de legrado y envío de la pieza para anális is histopatológico, resultando un quiste periapical. Conclusión: El diente 12 fue tratado endodónticamente después del diagnóstico y se está realizando un seguimiento de la paciente para analizar la formación de hueso sano en el lugar que anteriormente ocupaba el quiste.

Descriptores: Quiste Periapical; Necrosis de la Pulpa Dental; Maxilar.

INTRODUÇ̃̃O

O cisto periapical, também conhecido como cisto radicular ou cisto periodontal apical é um cisto odontogênico do tipo inflamatório, considerado, como um verdadeiro cisto revestido por epitélio que está associado ao epitélio do ápice de um dente não vital1.

Sua etiopatogenia está relacionada dos restos epiteliais de Malassez, epitélio crevicular, revestimento sinusal ou de revestimento epitelial 
dos tratos fistulosos e sua prevalência corresponde a cerca de $60 \%$ dos cistos da maxila e da mandíbula ${ }^{1,2}$.

O cisto periapical pode acontecer em qualquer idade; entretanto, acomete preferencialmente adultos, entre a terceira e a sexta décadas de vida ${ }^{2}$. O seu crescimento é lento e são assintomáticos ${ }^{3}$. A partir do momento de sua evolução, sem tratamento, pode atingir maiores dimensões, fazendo com que ocorra uma tumefação, sensibilidade, mobilidade e deslocamento dos dentes adjacentes ${ }^{2}$. Além disso, quando o cisto atinge grandes proporções pode causar dificuldade de abertura de boca, tumefação do maxilar envolvido e/ou desadaptação de próteses dentárias ${ }^{2,4}$.

O diagnóstico é feito através de exames radiográficos de rotina como uma radiografia periapical ou panorâmica. Lesões extensas em dentes com restaurações têm sido tratadas com sucesso, por meio do tratamento endodôntico conservador associado ao tratamento cirúrgico. A cirurgia periapical é indicada para lesões que excedem $2 \mathrm{~cm}$ e para aquelas associadas a dentes nos quais não é viável o tratamento endodôntico convencional ${ }^{2}$. Sempre após a cirurgia é indicado o exame histopatológico para descartar possíveis diagnósticos diferenciais, como o queratocisto odontogênico ${ }^{1}$.

O objetivo desse artigo é relatar um caso clínico de cisto periapical em região anterior da maxila com manejo cirúrgico e endodôntico.

\section{CASO CLÍNICO}

Paciente do sexo feminino, 20 anos de idade, foi encaminhada a clínica de Estomatologia do Centro de Pós-graduação em Odontologia, CPGO, com queixa de um discreto aumento de volume na região anterior da maxila (Figura 1).

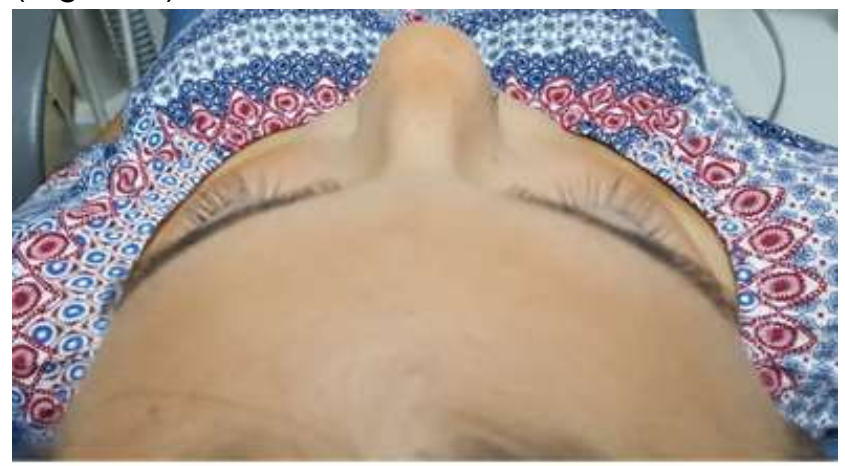

Figura 1: Vista superior da paciente com discreta assimetria do lado direito.

$\mathrm{Na}$ história médica, não apresentava nenhuma condição sistêmica digna de nota. Ao exame extraoral foi observada discreta assimetria e no exame intraoral, na região do dente 12 a mucosa apresentava-se com coloração normal e de consistência resiliente (Figura 2). Foi realizado teste de vitalidade no dente 12 que se mostrou negativo. A paciente não relatou nenhum histórico de trauma na região.

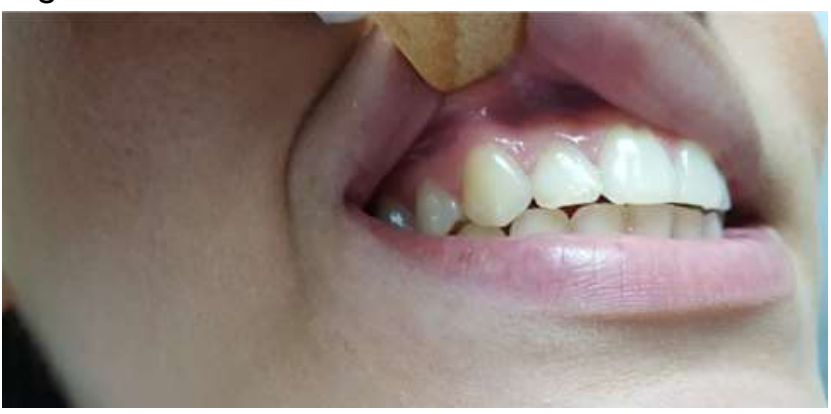

Figura 2: Aspecto intraoral do paciente.

A paciente trouxe à consulta radiografia e tomografia computadorizada, solicitado em seu último acompanhamento odontológico. No exame radiográfico foi observada imagem radiolúcida entre as raízes dos dentes 13 e 12 . O exame tomográfico confirmou a imagem com preservação da cortical óssea (Figura 3).

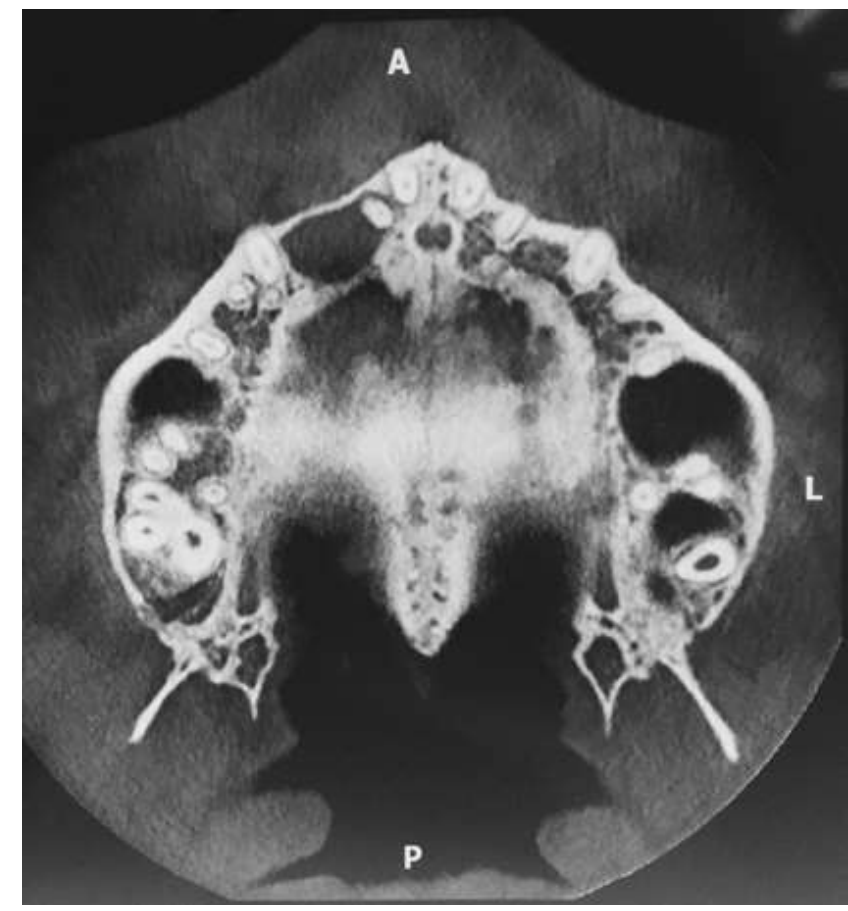

Figura 3: Corte Axial da Tomografia Computadorizada demonstrando lesão expansível na região de maxila com preservação da cortical óssea.

Frente aos aspectos clínicos e radiográficos, foi sugerida a hipótese diagnóstica de cisto periapical. Foi solicitado hemograma completo, coagulograma e glicemia que se apresentavam dentro dos padrões de normalidade. A conduta foi a exérese da lesão, seguidas de curetagem e envio do espécime para análise histopatológica e confirmar do diagnóstico presuntivo (Figuras 4 a 6). No exame histopatológico, observou-se a presença de epitélio estratificado pavimentoso revestindo uma cavidade cística e presença de tecido 
conjuntivo com moderado infiltrado inflamatório mononuclear e vasos sanguíneos hiperemiados, confirmando o diagnóstico de cisto periapical. A paciente foi encaminhada para o tratamento endodôntico do dente 12 e segue em acompanhamento.
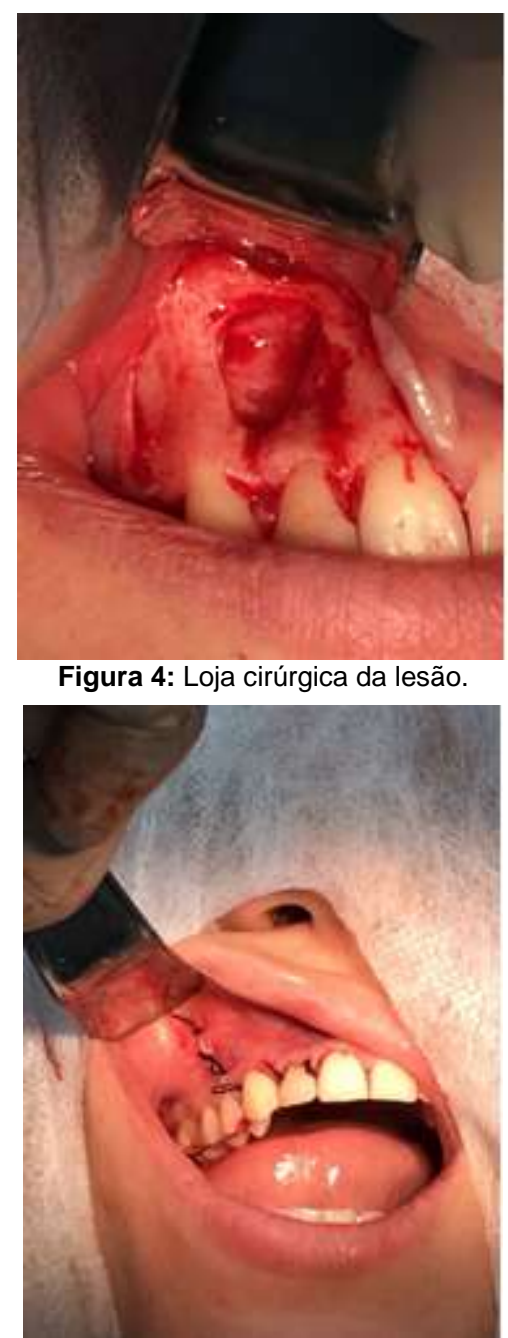

Figura 5: Aspecto final após remoção.

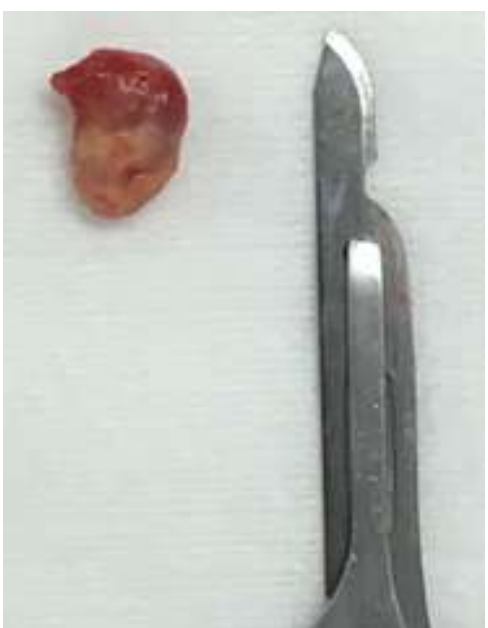

Figura 6: Espécime removido para análise histopatológica.

DISCUSSÃO

O cisto periapical é um do cisto odontogênico de origem inflamatória, sendo considerado uma lesão comum nos maxilares ${ }^{5}$. Sua causa está relacionada a necrose pulpar, seja por trauma ${ }^{1}$ ou por materiais restauradores com grandes extensões e próximos ao tecido pulpar sem a devida proteção ${ }^{2}$. O tratamento está relacionado à enucleação cirúrgica associada ao tratamento endodôntico do dente associado a lesão periapical.

Caracteristicamente, os pacientes com cistos periapicais não têm sintomas, a menos que haja uma exacerbação inflamatória aguda $^{6,7}$. O caso da paciente confirma esses achados, visto que apesar da evolução lenta da lesão não causava dor. Pelo fato de o cisto estar alcançando um tamanho grande, os primeiros sinais, como a tumefação, começaram a ser observados.

De acordo com Nobuhara e del Rio ${ }^{8}$, um diagnóstico clínico preliminar de cisto periapical pode ser feito, se tiverem as seguintes condições: 1) A lesão periapical envolver um ou mais dentes com necrose pulpar; 2) A lesão for maior do que $20 \mathrm{~mm}$ de diâmetro ou área transversal de $200 \mathrm{~mm}^{2}$; 3) cor palha no líquido de aspiração ou drenagem; 4) O fluido conter cristais de colesterol. No nosso caso o aspecto dos exames radiográficos e o teste de vitalidade com resposta negativa foi essencial para conduta do caso.

Estudos apontam que o movimento e a mobilidade dos dentes adjacentes são possíveis à medida que o cisto aumenta e que o dente no qual o cisto se originou não responde ao teste pulpar térmico e elétrico ${ }^{6,7}$. $\bigcirc$ padrão radiográfico é idêntico ao do granuloma periapical $^{1}$. Os cistos podem se desenvolver até mesmo em pequenas radiolucências periapicais e o tamanho radiográfico não pode ser utilizado para o diagnóstico definitivo ${ }^{1}$. Uma perda da lâmina dura é observada ao longo da raiz adjacente e uma radiolucidez arredondada cerca o ápice dentário afetado e a reabsorção radicular são comuns ${ }^{1,2}$.

As opções terapêuticas para as lesões císticas periapicais variam desde o tratamento não cirúrgico, correspondendo ao tratamento endodôntico convencional até 0 tratamento cirúrgico, como curetagem e enucleação cística. Existe também a possibilidade da variação dessas técnicas, como a descompressão e marsupialização prévia à enucleação, assim como a combinação das mesmas (cirurgia parendodôntica $)^{9,10}$. Os tratamentos cirúrgicos que podem ser realizados como alternativas em lesões radiculares extensas incluem a curetagem, enucleação, descompressão, marsupialização e ressecção em bloco ${ }^{11}$.

Os cistos de origem inflamatória não recidivam após um tratamento adequado e ocasionalmente podem ocorrer cicatrizes 
fibrosas em vez de neoformação óssea ${ }^{11} .0$ sucesso no tratamento de cistos periapicais de grande extensão pode ser alcançado através de um correto e minucioso diagnóstico, planejamento de tratamento adequado, acompanhamento clínico e radiográfico e cooperação do paciente ${ }^{9-11}$.

CONCLUSÃO

O tratamento de cisto periapical deve ser realizado de maneira eficaz para evitar recidivas da lesão. O exame clínico completo e minucioso da cavidade bucal onde o cirurgião-dentista colhe dados de histórico da doença são necessários para 0 correto diagnóstico e tratamento de lesões na região dos maxilares.

\section{REFERÊNCIAS}

1. Neville BW, Damm DD, Allen CM, Bouquot JE. Patologia oral e maxilofacial. 4. ed. Rio de Janeiro: Guanabara koogan; 2016.

2. Pereira JF, Milagres RM, Andrade BAB, Messora MR, Kawata LT. Extensive Radicular Cyst: a Case Report. Rev cir traumatol bucomaxilo-fac. 2012;12(2):37-42.

3. Garcia de Mendonça JC, Gaetti Jardim EC, Santos CM, Masocatto DC, Quadros DC, Oliveira MM, Macena JÁ, Teixeira FR. Cisto periapical residual: relato de caso clínicocirúrgico. Arch Health Invest. 2015;4(1):45-9.

4. Corrêa M, Elias R, Cherubim K, Ponzoni D. Cisto Radicular Residual: Relato de Caso Clínico. J bras clín odontol integr. 2002;6(32): 133-35.

5. Oliveira DHIP, Lima ENA, Araújo CRF, Germano AR, Medeiros AMC, Queiroz, LMG. Cisto residual com grande dimensão: relato de caso e revisão da literatura. Rev cir traumatol buco-maxilo-fac. 2011;11(2):21-6.

6. Aggarwal V, Singla M. Use of computed tomography scans and ultrasound in differential diagnosis and evaluation of non-surgical management of periapical lesions. Endodontol. 2010;109(6):917-23.

7. Graziani M; Cirurgia bucomaxilofacial. Rio de Janeiro: Guanabara Koogan; 1995

8. Nobuhara W, Del Rio C. Incidence of periradicular panthoses in endodontic treatment failures. J Endod. 1993;19(6):315-18.

9. Peker E, Ogutlu F, Karaca IR, Gultekin ES, Cakir M. 5 year retrospective study of biopsied jaw lesions with the assessment of concordance between clinical and histopathological diagnoses. J Oral Maxillofac Pathol. 2016;20(1):78-85.

10. Bava FA, Umar D, Bahseer B, Baroudi K. Bilateral radicular cyst in mandible: an unusual case report. J Inte Oral Health. 2015;7(2)61-3.
11. Resende MAP, Assis NMSP, Sette-Dias AC, Aguiar EG, Sotto-Maior BS. Tratamento cirúrgico e conservador de cisto periapical de grande proporção: relato de caso. HU Revista. 2017;43(2):191-96.

\section{CONFLITO DE INTERESSES}

Os autores declaram não haver conflitos de interesse

AUTOR PARA CORRESPONDÊNCIA

\section{Híttalo Carlos Rodrigues de Almeida}

Instituto de Tecnologia de Pernambuco - ITEP/Bloco B 10. andar

Faculdade de Odontologia da Universidade de Pernambuco

Av. Prof. Luís Freire, 700 - Cidade Universitária,

50740-540 Recife-PE, Brasil

E-mail: hittalo.rodrigues@upe.br 